\title{
A Sub-cell Based Channel Reservation Method for MEO Network
}

\author{
Luoyu Qin ${ }^{1}$, Ying Tao ${ }^{1}$ and Hui $\mathrm{He}^{2+}$ \\ ${ }^{1}$ China Academy of Space Technology, Beijing, China \\ ${ }^{2}$ Beijing Institute of Technology, Beijing, China
}

\begin{abstract}
Medium Earth Orbit (MEO) satellite networks must be capable of handle the handover problem of users between different spot-beam cells. In this paper, a Sub-cell based Handover Scheme (SHS) is proposed to decrease the resource reservation time reasonably in the Dynamic Doppler-Based Handover Prioritization (DDBHP) scheme. In this way, we decrease the new call blocking probability effectively while keeping very small of the forced termination probability for the handover user, which greatly increases the resource utility. Simulation results verify the correctness of the proposed approach.
\end{abstract}

Keywords: MEO satellite networks; handover management; time-based reservation.

\section{Introduction}

Handover scheme for satellite networks has been thoroughly researched nowadays. Most of them are based on the way of resource reservation, that is, the resources are reserved in advance for users to guarantee the success of handover. Guarantee Handover $(\mathrm{GH})$ method [1], as the pioneer of resource reservation schemes, tries to reserve a channel in the cell next to the one the user is entering. However, GH is somewhat selfish for its over conservative behaviour, in which locked channel cannot be used by other users, even though the owner does not use it currently. That is why the DDBHP scheme [2]-[4] is proposed to lock the resources only for their expected handover time of use for a better channel utilization. By anticipating the user motion and reserving resources, DDBHP estimates the residence time of the user in each cell to be crossed and reserves a resource during the corresponding residence time interval. Similarly, other solutions have been provided to delay the channel locking in the next candidate cell and trade-off the handover guarantee to a certain extent: The Elastic Channel Locking scheme [5], Time-based Channel Reservation Algorithm method [6][7], Threshold-Based Handover Prioritization scheme [8], Channel Status based Reservation Strategy [9], Dynamic Channel Reservation scheme based on Priorities [10], and a geographical information based resource reservation algorithm [11].

State-of-the-art methods are devoted in delaying the reservation request moment as much as possible while ensuring the QoS of the UT. Nevertheless, they usually set the time reserved in the next spot-beam cell for the UT as the channel duration of a spot-beam cell. This kind of resource reservation methods has a certain applicability in LEO networks with short channel duration of the spot-beam cell. However, the channel duration of the spot-beam cell is longer in MEO network. To clarify this problem, assume that the constellation of a satellite network is composed of five MEO satellites, with the height of $10390 \mathrm{~km}$ and $0^{\circ}$ inclination, which meets the seamless communication requirement for low latitude area. The duration of a spot-beam cell is about 10 minutes, while a typical voice call usually lasts about 3 minutes. With the state-ofthe-art methods applied, 10 minutes channel resource would be reserved for the UT whose average demand is just 3 minutes. Apparently, there is a significant waste of resources in these methods. In this paper, we propose a Sub-cell based Handover Scheme (SHS) based on DDBHP to provide a better resource utilization

+ Corresponding author. Tel.: +8618612449131

E-mail address: qinluoyu@icloud.com 
of the MEO system. In this scheme, the duration of channels locked is equal to the expected communication time of the UT. This methodology allows to perform more accurate reservations in order to increase the number of admitted users in the system.

The remaining of this paper is organized as follows: In section 2, we briefly descript the DDBHP handover scheme and shows how our proposed SHS method improves the DDBHP performance. An analytical approach is developed in Section 3 to evaluate the performance of SHS algorithm. In Section 4, simulation experiments are derived to validate the results obtained analytically. Finally, the conclusions are reported in Section 5.

\section{Resource Reservation Based Handover Problem in MEO Satellite Network}

In this paper, we focus on the Satellite-Fixed Cells (SFC) and Fixed Channel Allocation (FCA) scheme [2]. To clarify the differences between SHS and DDBHP scheme, the user relative mobility models of DDBHP and SHS are illustrated in Fig. 1 and Fig. 2 respectively.

In the DDBHP scheme, $T_{\text {cell }}$ denotes the duration of a spot-beam cell, the time threshold $t_{T H}$ indicates the moment when the reservation request is sent. The properly choosing $t_{\mathrm{TH}}$ allows DDBHP to achieve different levels of forced termination probability $P_{f}$ or even eliminate it, because whether or not a new call is admitted in the network depends on the position of the UT at the call setup:

- If the UT is located in region A, an available channel only in the present spot-beam cell is required;

- If in region $\mathrm{B}$, available channels both in the present and the next spot-beam cell are required.

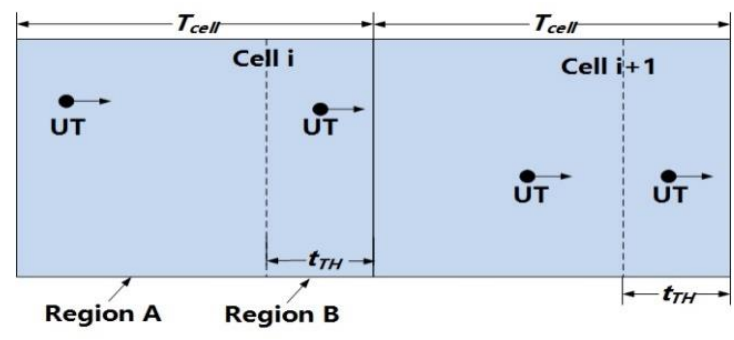

Fig. 1: User relative mobility model in DDBHP

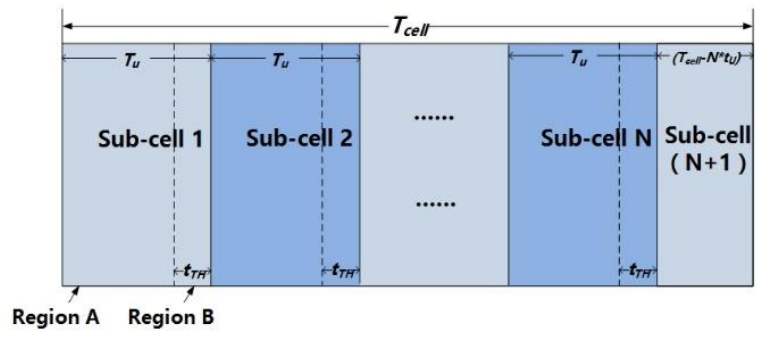

Fig. 2: The sub-cell model of spot-beam cell

However, there are two main differences between SHS and DDBHP scheme: the channel reserved time for the UT and the time threshold $t_{T H}$. Since the channel duration $T_{c e l l}$ of the spot-beam cell is much longer than the average communication time $\mathrm{T}_{\mathrm{u}}$ of the UT in MEO networks. As shown in Figure 2, the spot-beam cell is divided into multiple sub-cells to avoid the excessive resource reservation in SHS. Because of the noninteger of $\frac{T_{c e l l}}{T_{u}}$, the channel duration of the $(N+1)_{t h}$ sub-cell is $\left(T_{\text {cell }}-N * T_{u}\right)$, where $N=\left\lfloor\frac{T_{c e l l}}{T_{u}}\right\rfloor$. Thus, the resource reservation time for the UT is the channel duration $t_{R}^{i}$ of the $i_{t h}$ sub-cell in SHS, instead of the channel duration of a whole spot-beam cell $T_{\text {cell }}$ in DDBHP. In addition, different from DDBHP scheme, the time threshold $t_{T H}$ divides a sub-cell into region A and region B in SHS. If the UT is located in region A of a sub-cell, an available channel only in the present sub-cell is required. If the UT is located in region B of a sub-cell, available channels both in the present and in the next sub-cell are required. In both case, similar to DDBHP, if the next sub-cell has an available channel, it is locked for the UT immediately. Otherwise, the reservation request is queued for a time interval. If a channel is not found during the time $t_{T H}$, the communication of the UT is forced into termination. If the communication is terminated before the handover occurrence, the reserved channel or the queued request are also cleared.

\section{Analytical Approach for SHS}

In this process, we analyze the performances of forced termination probability $P_{f s}$ and blocking probability $P_{b s}$ in a sub-cell firstly. Then the mathematical analysis of $P_{f}$ and $P_{b}$ are extended. We consider the following basic assumptions, also as usually made in the literature [1], [2], [5], [10]:

- The new call and handover arrival processes of UTs are independent Poisson processes;

- The distribution of UTs in spot-beam cells are uniform;

- The communication times of UTs are exponentially distributed, with mean value $T_{u}$. 


\subsection{The performance analysis in a sub-cell}

According to equilibrium condition [12], the equilibrium equation in region $\mathrm{A}$ and $\mathrm{B}$ of a sub-cell can be derived as

$$
\begin{aligned}
& \lambda_{r}=\lambda_{h} P_{h 2-A}+\lambda_{n-A}\left(1-P_{b s}\right) P_{h 1-A} \\
& \lambda_{r}\left(1-P_{f s}\right) P_{h 2-B}+\lambda_{n-B}\left(1-P_{b s}\right)^{2} P_{h 1-B}=\lambda_{h}
\end{aligned}
$$

where $\lambda_{h}$ denotes the arrival rate of handover calls, $\lambda_{r}$ is the rate of handover requests, and $\lambda_{n-A}, \lambda_{n-B}$ represent the arrival rate of new calls in regions A and B. Similar to literature [2], $P_{h 1-A}=\alpha_{A}\left(1-e^{-\frac{1}{\alpha_{A}}}\right)$, $P_{h 1-B}=\alpha_{B}\left(1-e^{-\frac{1}{\alpha_{B}}}\right)$ denote the first handover in region A and B, $P_{h 2-A}=e^{-\frac{1}{\alpha_{A}}}, P_{h 2-B}=e^{-\frac{1}{\alpha_{B}}}$ denote the subsequent handover in region $\mathrm{A}$ and $\mathrm{B}$, where dimensionless parameters $\alpha_{A}=\frac{T_{u}}{t_{R}-t_{T H}}, \alpha_{B}=\frac{T_{u}}{t_{T H}}$, and traffic parameters $\lambda_{n-A}=\frac{\lambda_{n}}{\alpha_{A}}, \quad \lambda_{n-B}=\frac{\lambda_{n}}{\alpha_{B}}$.

There are four kinds of UTs who occupying resources in current sub-cell: 1) In the current sub-cell, the new arrival UTs generated in region A; 2) In the current sub-cell, the new arrival UTs generated in region B; 3) In the previous sub-cell, the new arrival UTs generated in region B need reservation in the current sub-cell; 4) In the previous sub-cell, the UTs reaching the boundary $t_{T H}$ of region A and B need resource reservation in the current sub-cell.

Similar with literature [2], the derivation of the average channel holding time $\frac{1}{\mu}=\sum_{n=1}^{4} P_{n} E\left[T_{H}^{n}\right]$ for UTs in a sub-cell consists of four situations above, each of which represents a different case of call. When the number of occupied channels in the sub-cell is less than $C$, all these four kinds of UTs above can occupy channels, and the channel occupancy rate of the sub-cell $\lambda_{s p}$ is

$$
\lambda_{s p}=\lambda_{n-A}+\lambda_{n-B}\left(1-P_{b s}\right)+\lambda_{n-B}\left(1-P_{b s}\right)+\lambda_{r}
$$

Thus, the Markov chain model for SHS can be depicted in Fig. 3. Each state represents the sum of the channel in service and reservation request in the waiting queue. For $k \in[0, C-1]$, the transition rate from state $k$ to $k+1$ is given by $\lambda_{s p}$ in (3). However, for $k \in[C, 2 C-1]$, because all channels are busy, the arriving new calls would be discarded immediately and only the fourth kind of UTs above (who requesting resource reservation) would be queued to wait for a chance, so the transition rate from state $k$ to $k+1$ is $\lambda_{r}$. The parameter $\mu_{u t}$ is the average departure rate of UTs, which meets $\mu_{u t}=\frac{1}{T_{u}}$.

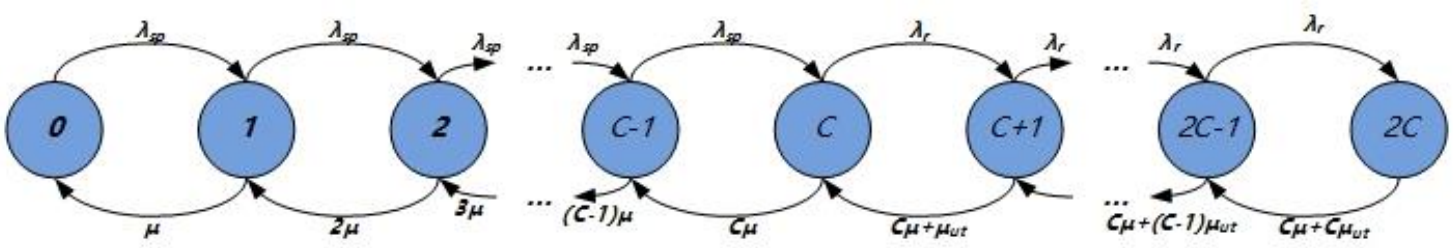

Fig. 3: Markov chain model for SHS

Similar with DDBHP scheme [4], $P_{s}(k)$ (the steady-state probability of state $k$ ) is derived

$$
P_{S}(k)= \begin{cases}\frac{\left(\lambda_{s p}\right)^{k}}{k ! \mu^{k}} P_{S}(0), & 0 \leq k<C \\ \frac{\left(\lambda_{s p}\right)^{C}\left(\lambda_{r q}\right)^{(k-C)}}{C ! \mu^{C} \prod_{j=1}^{k-C}\left(C \mu+j \mu_{u t}\right)} P_{S}(0), & C \leq k \leq 2 C\end{cases}
$$

where the channel idle probability $P_{S}(0)$ is given by

$$
P_{S}(0)=\left\{\sum_{s=0}^{C-1} \frac{\left(\lambda_{s p}\right)^{k}}{k ! \mu^{k}}+\sum_{s=C}^{2 C} \frac{\left(\lambda_{s p}\right)^{C}\left(\lambda_{r q}\right)^{(k-C)}}{C ! \mu^{C} \prod_{j=1}^{k-C}\left(C \mu+j \mu_{u t}\right)}\right\}^{-1}
$$


Therefore, the block probability $P_{b s}$ and forced termination probability $P_{f s}$ of a sub-cell can be derived as

$$
\begin{aligned}
& P_{b s}=\sum_{k=C}^{2 C} P_{S}(k) \\
& P_{f S}=\sum_{k=C}^{2 C} P_{S}(k) \operatorname{Pr}\left\{t_{q} \geq t_{T H}\right\}=P_{n s} e^{\left(\lambda_{r q}-C \mu\right) t_{T H}}
\end{aligned}
$$

\subsection{The performance analysis in a spot-beam cell}

As the sub-cell is divided from a spot-beam cell (Fig. 2), we set $t_{R}^{i}$ be the channel duration of $i_{t h}$ sub-cell, and $P_{b s}^{i}, P_{f s}^{i}$ be the block probability and forced termination probability respectively. The block probability $P_{b}$ and forces termination probability $P_{f}$ of a spot-beam cell can be deduced as

$$
\begin{aligned}
& P_{b}=\sum_{i=1}^{N+1} \frac{t_{R}^{i}}{T_{c e l l}} P_{b s}^{i} \\
& \left.P_{f}=\sum_{m=1}^{\infty}\left(1-\left(1-P_{f s}\right)^{m}\right) P\left\{(m-1) T_{u}<t_{u}<m T_{u}\right\}\right)=\sum_{m=1}^{\infty}\left(1-\left(1-P_{f s}\right)^{m}\right) \frac{e-1}{e^{m}}=\frac{e P_{f s}}{e-\left(1-P_{f s}\right)}
\end{aligned}
$$

Based on the analysis provided in this section, with the help of recursive approach, $t_{T H}$ can be calculated for meeting the required performance in terms of $P_{b}$ and $P_{f}$.

\section{Simulation and Analytic Results}

The purpose of this section is to verify the validity of SHS method by comparing the simulation results with DDBHP scheme. In obtaining our simulation, we use the mobility model shown in Section 2. Each spot-beam cell has 10 channels. The channel duration of a spot-beam cell $T_{\text {cell }}$ equals to 360 seconds, which is calculated according to the satellite altitude and the cell size of ICO system [13]. In this system, we implemented a Poisson procedure for arriving traffic by using a number of UTs $N_{U T S}=1000 \gg C$ and the traffic load ranging from 6 to 24 Erlang. 1200 UTs were uniformly distributed in each spot-beam cell, and the communication time $t_{u}$ of the UT is exponentially distributed with the mean value set to $T_{u}=180 \mathrm{~s}$. Queuing policy for channel resource reservation request is First In First Out (FIFO). Thus, the number of sub-cells for a spot-beam cell is 2 in SHS, and the channel duration of a sub-cell $t_{R}=180 \mathrm{~s}$.

When the cell load is 24 Erlang in system, the Blocking probability $P_{b}$ and forced termination probability $P_{f}$ versus threshold $t_{T H}$ in SHS is shown in Fig. 4. Apparently, $P_{f}$ in SHS is actually zero when $t_{T H} \geq 0.4 t_{R}$, and the $P_{b}$ would increase with the higher of $t_{T H}$. Therefore, on the basis of satisfying the QoS demand of the UT on $P_{f}$, we should decrease the value of $t_{T H}$ as much as possible to improve the system resource utilization.

The comparison on the blocking probability $P_{b}$ and forced termination $P_{f}$ of SHS and DDBHP method is shown in Fig. 5, where the cell load still be 24 Erlang. Because the reservation time for UTs are determined by the average duration of communication $T_{u}$ in SHS, rather than the channel duration $T_{\text {cell }}$ of a spot-beam cell in DDBHP, the wasted reserved resources which cannot be used by other users is significantly reduced in SHS. Thus, the $\mathrm{P}_{\mathrm{b}}$ in SHS keeps obviously lower than that of DDBHP. However, because the resource reservation time $T_{u}$ in SHS algorithm is shorter than that of DDBHP (which is $T_{\text {cell }}$ ), the UT with a communication time longer than $T_{u}$ has to handover again in SHS scheme, which increases the number of handover in SHS. As a result, the performance on $P_{f}$ is slightly worse in SHS than that of DDBHP scheme. Nevertheless, if we choose the $t_{T H}$ appropriately and keep the $P_{f}$ in very small value in SHS, the negative effect of worse performance on $P_{f}$ can be eliminated.

Fig. 6 shows the blocking probabilities $P_{b}$ of SHS and DDBHP under different cell load versus reservation threshold $t_{T H}$, and Fig. 7 depicts the blocking probabilities $P_{b}$ of SHS and DDBHP under different reservation threshold $t_{T H}$ versus cell load. Both of them demonstrated the validity that the proposed SHS method can inherit the advantage of DDBHP method, while making a better resource utilization in MEO satellite networks. 


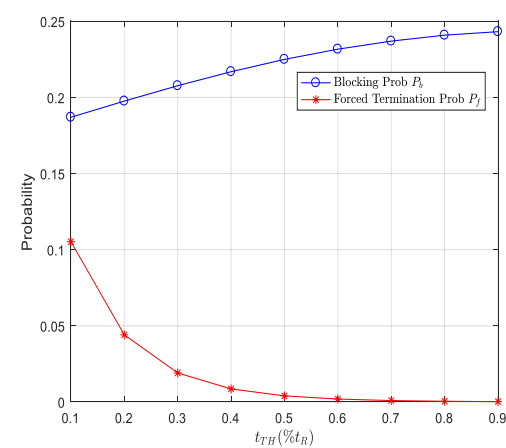

Fig. 4: Blocking probability and forced termination probability versus threshold in SHS

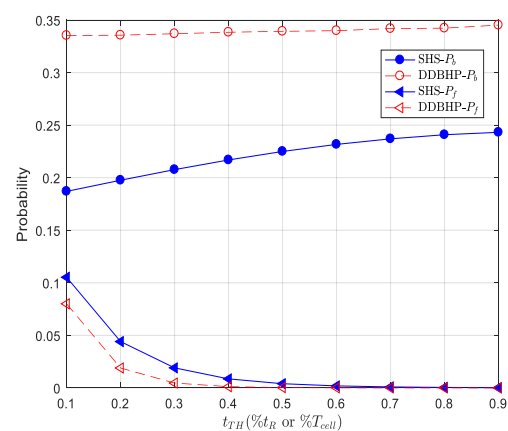

Fig.5: Blocking probability and forced termination probability of SHS and DDBHP versus threshold

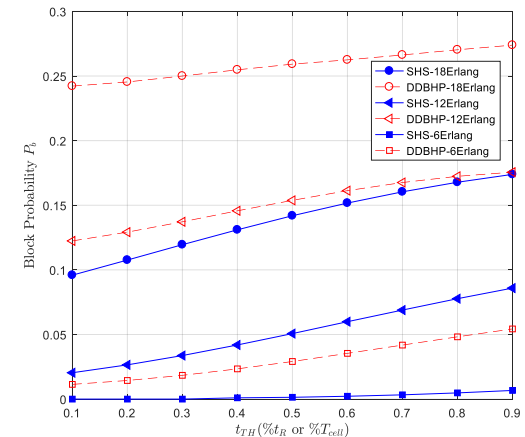

Fig. 6: Blocking probability of SHS and DDBHP under different cell load versus threshold

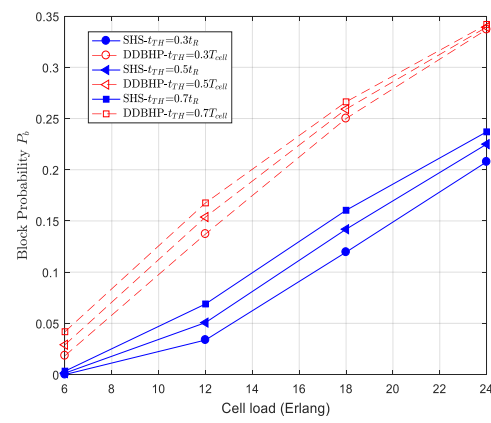

Fig.7: Blocking probability of SHS and DDBHP under different threshold versus cell load

\section{Conclusions}

In this paper, we point out the problem of over-reservation in the resource reservation based handover methods, which has never been addressed so far in contributions. And a sub-cell based handover scheme was proposed, which takes the advantage of the statistical communication time of the traffic, to solve the overreservation problem for a better resource utilization. Simulation results show that the proposed method can satisfy QoS demands of users on the forced termination probability while achieving better blocking probabilities.

\section{Acknowledgment}

This work was supported by the National Natural Science Foundation of China (No.91438205).

\section{References}

[1] G. Maral, J. Restrepo, E. Del Re, R. Fantacci, and G. Giambene, "Performance analysis for a guaranteed handover service in an leo constellation with a" satellite-fixed cell" system," IEEE Transactions on Vehicular Technology, vol. 47, no. 4, pp. 1200-1214, 1998.

[2] E. Papapetrou and F.-N. Pavlidou, "Analytic study of doppler-based handover management in leo satellite systems," IEEE Transactions on Aerospace and Electronic Systems, vol. 41, no. 3, pp. 830-839, 2005.

[3] E. Papapetrou and F.-N. Pavlidou, "Qos handover management in leo/meo satellite systems," Wireless Personal Communications, vol. 24, no. 2, pp. 189-204, 2003.

[4] S. Karapantazis and F.-N. Pavlidou, "Design issues and qos handover management for broadband leo satellite systems," IEE Proceedings- Communications, vol. 152, no. 6, pp. 1006-1014, 2005.

[5] Y. Xu, Q. L. Ding, and C. C. Ko, "An elastic handover scheme for leo satellite mobile communication systems," in Global Telecommunications Conference, 2000. GLOBECOM'00. IEEE, vol. 2. IEEE, 2000, pp. 1161-1165.

[6] L. Boukhatem, A.-L. Beylot, D. Ga" iti, and G. Pujolle, "Tcra: a time- based channel reservation scheme for 
handover requests in leo satellite systems," International Journal of Satellite Communications and Net- working, vol. 21, no. 3, pp. 227-240, 2003.

[7] L. Boukhatem, D. Gaiti, and G. Pujolle, "Resource reservation schemes for handover issue in leo satellite systems," in Wireless Personal Mul- timedia Communications, 2002. The 5th International Symposium on, vol. 3. IEEE, 2002, pp. 1217-1221.

[8] D. Ding, D.-T. Ma, and J.-B. Wei, "A threshold-based handover priori- tization scheme in leo satellite networks," in Wireless Communications, Networking and Mobile Computing, 2008. WiCOM'08. 4th International Conference on. IEEE, 2008, pp. 1-6.

[9] X. Wang and X. Wang, "The research of channel reservation strategy in leo satellite network," in Dependable, Autonomic and Secure Computing (DASC), 2013 IEEE 11th International Conference on. IEEE, 2013, pp. 590594.

[10] J. Zhou, X. Ye, Y. Pan, F. Xiao, and L. Sun, "Dynamic channel reservation scheme based on priorities in leo satellite systems," Journal of Systems Engineering and Electronics, vol. 26, no. 1, pp. 1-9, 2015.

[11] C. Pan, H. Luo, L. Yang, and D. Wei, "Leo satellite communication system handover technology and channel allocation strategy," Interna- tional Journal of Innovative Computing, Information and Control, vol. 9, no. 11, pp. 4595-4602, 2013.

[12] E. Del Re, R. Fantacci, and G. Giambene, "Characterization of user mobility in low earth orbit mobile satellite systems," Wireless Networks, vol. 6, no. 3, pp. 165-179, 2000.

[13] F. Makita and K. Smith, "Design and implementation of ico system," in 17th AIAA International Communications Satellite Systems Conference and Exhibit, 1998, p. 1216. 